\title{
Design and Simulation of EV Charging Device Based on Constant Voltage-Constant Current PFC Double Closed-Loop Controller
}

\author{
Cheng Zhang ${ }^{1,}$ a, Yihui Zheng ${ }^{1, b^{*}}$, Lixue $\mathrm{Li}^{1}$, Xin Wang ${ }^{1}$, Jianyou $\mathrm{Yu}^{2}$, Jingbo \\ Yang $^{2}$
}

${ }^{1}$ Center of Electrical \& Electronic Technology, Shanghai Jiao Tong University, Shanghai, China

${ }^{2}$ Baishan Power Supply Company of State Grid Jilin Electric Power Company, Baishan, China a934375200@qq.com, ${ }^{b}$ zhengyihui@sjtu.edu.cn

"corresponding author: zhengyihui@sjtu.edu.cn

Keywords: Electrical vehicles; Constant voltage-constant current charging; Dual closed-loop control; PFC.

Abstract. Electrical vehicle has low emissions, high efficiency and other merits. In this paper, an efficient charging device program based on constant current-constant voltage charging, PFC, and Dual Closed-Loop control is proposed. Firstly, at the beginning of the charging stage, constant current charging method can keep the charging current from being too large. When the charge process comes to an end, constant voltage charging method can avoid the battery over charge.So, a constant current-constant voltage charging method is used to guarantee both the charging time and the battery working condition. And then, half-bridge isolated DC-DC converter which can achieve the function of electrical isolation and circuit switch control is presented. Secondly, to improve the charging efficiency, the main circuit takes advantage of Dual Closed-Loop control. Based on the output side of the current and voltage sampling, the information is fed back to the switch. Thirdly, PFC is added to the main circuit. It can improve the power factor of the circuit and reduce the harmonic distortion of the input current, so that the harmonic pollution caused by the charging process can be reduced. Finally, in the PSIM simulation and research on the main circuit, the simulation results show that the charging scheme has the good value of application.

\section{Introduction}

As energy shortage and air pollution increasing, electrical vehicle with low emissions, high efficiency and other characteristics, has become the important breakthrough of the automobile industry to energy security, climate change and energy structure upgrade issues [1].

Scholars in China and abroad have carried out a series of constructive study in terms of increasing the benefit of the electrical vehicle charging. In [2], it proves that the constant pressure-constant current charging way is more in line with the actual battery charging process. The literature [3] proves that to maximize the efficiency in the process of electrical vehicle charging, we can control the switching frequency of the charging device to achieve the desired effect. In [4], it can be found that to improve charging efficiency, we can improve the power factor of the charging device and reduce the harmonic distortion from current and voltage. We can find it from the literature [5] that the EV will make losses of the power distribution network, influence the balance of the network and also shorten the equipments' life.

Unlike the previous charging devices, this article will comprehensively consider the charging efficiency of the battery and the influence of charging process on the power grid. We apply constant pressure-constant current charging, PFC, and double closed-loop control strategy to the charging device. Constant current-constant voltage charging can guarantee the charge time and make the battery work normally. Based on the output side of the current and voltage sampling, Dual Closed-Loop control can improve the charging efficiency. Besides, PFC can reduce the harmonic pollution caused by the charging process. In the sections below, the control algorithm and the technique of charging will be taken into consideration. 


\section{Charging Technology}

In order to realize good performance of the electrical vehicle charging, we have to seek the best charging mode and increase the speed of charging to ensure that electrical vehicle can work safely and efficiently. Next the charging scheme is discussed in the section below.

Constant Voltage-Constant Current Charging. Constant voltage-constant current charging combine the constant voltage charging method and constant current charging method together. At the beginning of the charging stage, it uses the constant current charging to keep the charge current from being too large. When the charging process comes to the end, constant voltage charge can avoid the battery over charge. So we adopt these two charging methods to achieve good working condition.

\section{Charging Device Related Parameter Design}

For the charging unit, the input voltage is $220 \mathrm{~V}$ (AC), and the output voltage is controlled in 260-340 $\mathrm{V}$ (DC). The maximum output current is at about $8 \mathrm{~A}$. In the device, the filter inductance is $27 \mu \mathrm{H}$, and filter capacitance is $22 \mu \mathrm{F}$.

Selection of the Capacitor. The choice of capacitance is critical. Through the analysis of circuit, the capacitance can be concluded as.

$$
C_{f}=\frac{V_{o}}{8 L_{f} f_{\text {ef }} \Delta V_{o p p}}\left(1-\frac{V_{0}}{V_{\text {in }}-V_{L P}-V_{o}}\right) .
$$

So the size of the capacitance can be chosen as $22 \mu \mathrm{F}$.

The Selection of the Inductance. In order to reduce the disturbance of the circuit, it needs to use the inductance. The calculation of filter inductance is as follows :

$$
\left.L_{f}=\frac{V_{o}}{2\left(2 f_{s}\right) \times\left(10 \% I_{o(\max }\right)}\right)\left[1-\frac{V o(\max )}{\frac{V i n(\max )}{K}-V_{4}-V_{D}}\right] .
$$

Here select the inductance roughly at about $27 \mu \mathrm{H}$ by the calculation.

The Selection of the DC-DC converter. Switching power supply topology structure can be divided into two major categories: the isolation and the non isolation converter. The transformer plays an important role in the electrical isolation, which isolates input and output circuit. This structure can adjust the size of the output voltage. The number of the switch device in the half bridge circuit is less than that of the whole bridge. On the condition of the same power, the cost of the half bridge circuit is lower. Half bridge circuit also has another advantage that it can effectively prevent the phenomenon of the magnetic field deviation. So in this paper, the half bridge non isolation DC- DC conversion circuit is adopted.

\section{The Control Technology}

Dual-Loop Control. This paper uses double closed-loop circuit to control the output current and voltage. The double closed-loop control principle is shown in Fig. 1. 


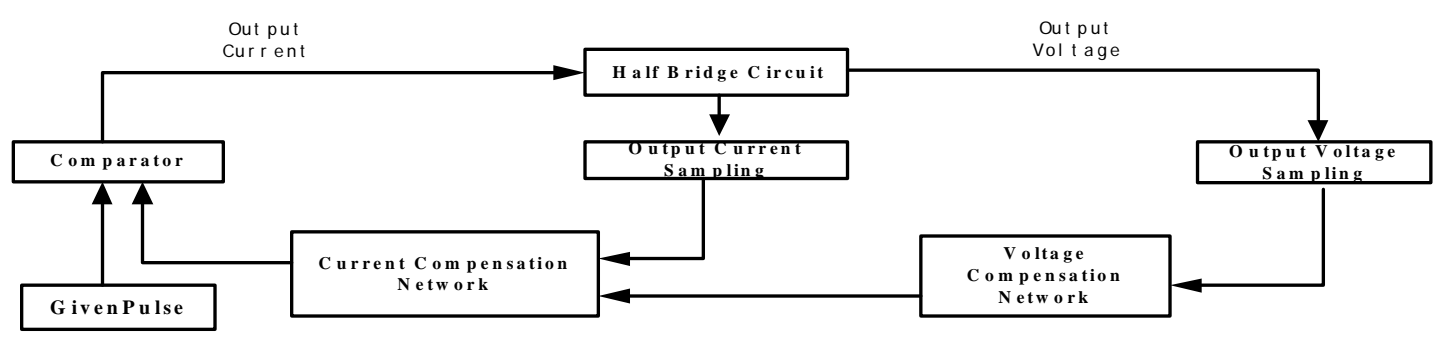

Fig. 1 Double closed-loop structure

According to the figure, double closed-loop control can be divided into the current loop and voltage loop. The current compensation loop and voltage compensation loop are controlled by the PI regulator. In this paper, it uses continuous system design method to determine the PI parameters. To apply it to the digital control system, the system control equations need to be discrete. Current loop and voltage loop control chart is shown in Fig. 2.

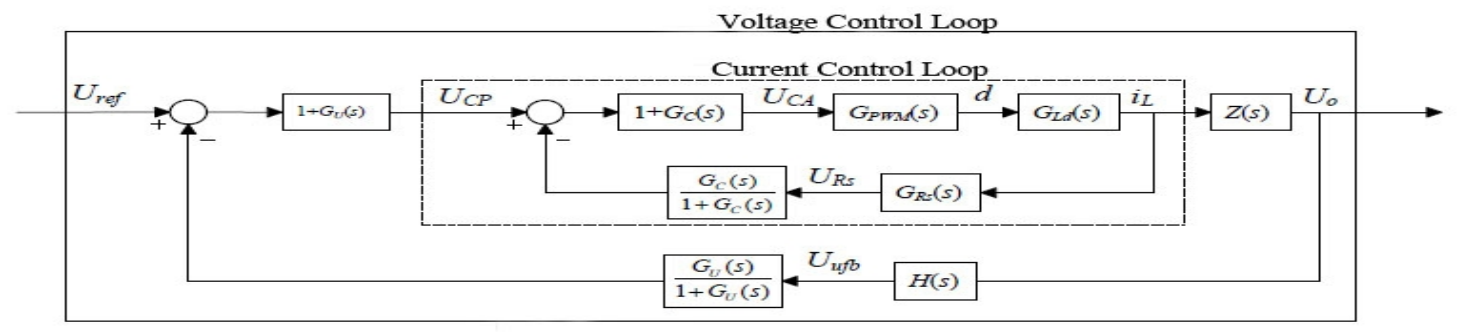

Fig. 2 Control chart of double closed loop system

Power Factor Correction. Power factor correction circuit compares the output voltage and the given voltage and gives the related information to the voltage regulator. The output voltage is multiplied by the voltage of the sine wave and the instruction current can be got. Then the instruction current and the actual current are compared through the subtraction. Through a series of control steps in the switch, the current and voltage can be close to the same phase of the sine wave.

\section{Simulation and Result Analysis of Charging Device}

Simulation. The main circuit model is shown in Fig. 3.

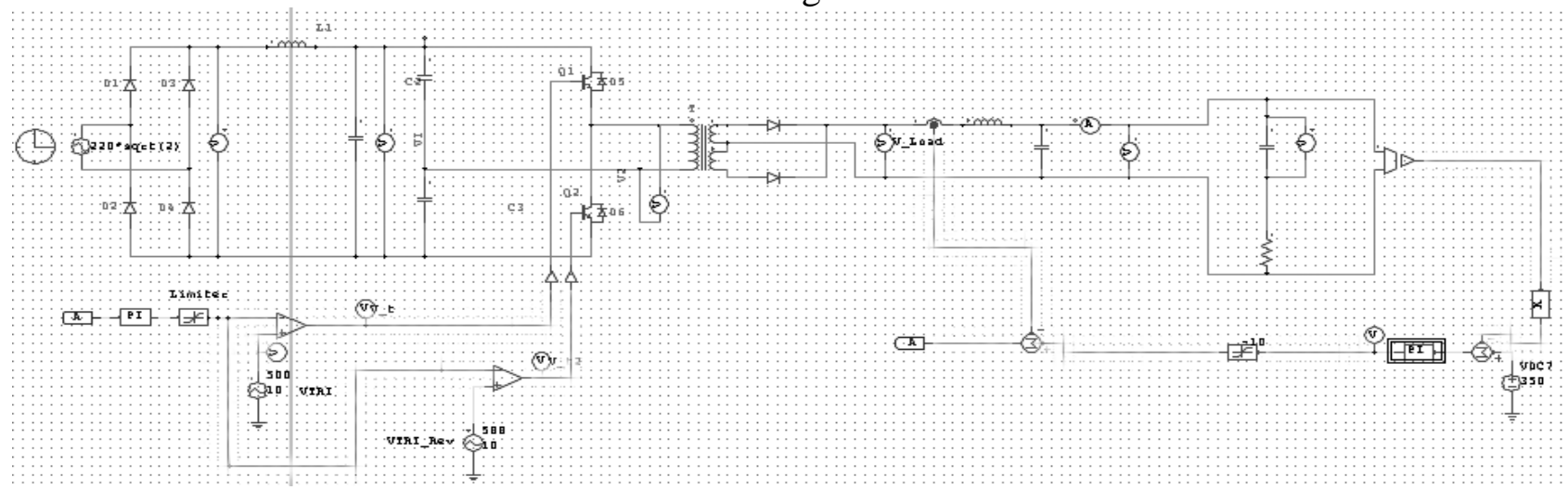

Fig. 3 Main circuit structure

It can be seen that the output voltage is at about $340 \mathrm{~V}$ which reaches the maximum charge voltage of the battery. The voltage waveform of the charging device is shown in Fig. 4. 


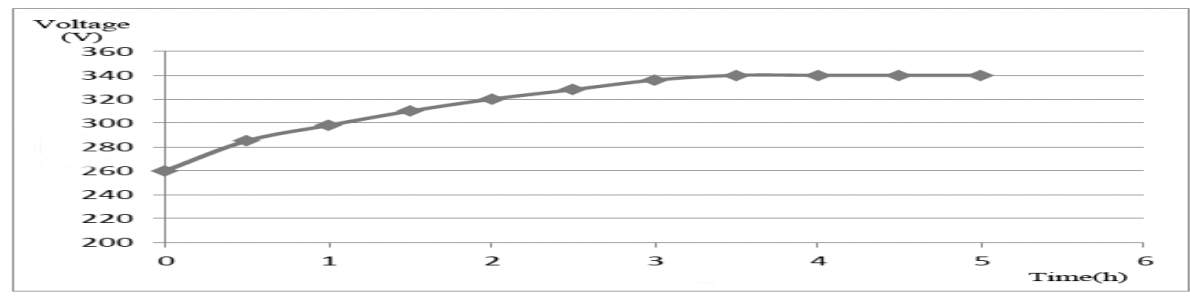

Fig. 4 Voltage change

The current transformation graph is shown in Fig. 5.

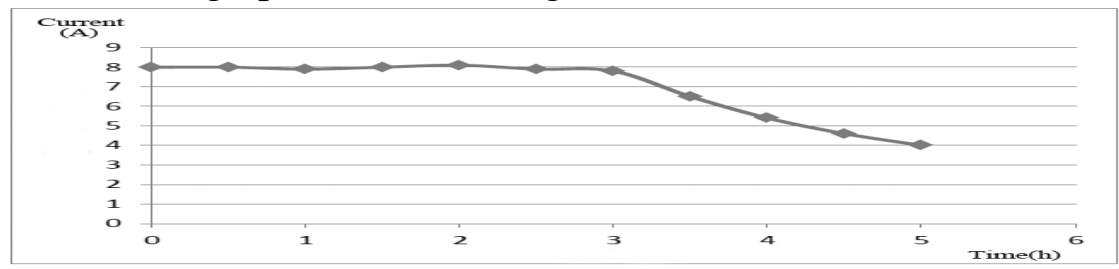

Fig. 5 Current change

Simulation Result Analysis. Through the above two graphics, it can be found that in the process of charging, as the battery absorbs power, the battery voltage is increasing. When the voltage reaches about $340 \mathrm{~V}$, the charging process is approximately stable. When the current reaches about $8 \mathrm{~A}$, the charging process gradually remains stable. The variation of voltage and current is in accordance with the requirements of constant current- constant voltage charging. It takes about 5 hours for the circuit to complete the charging process which is much shorter than the time of charging pole scheme. Through the analysis of voltage and current changes, it can be found that PFC has effectively reduced the power grid pollution in the charging process.

\section{Conclusions}

In this paper, an intelligent charging device for the electrical vehicle is designed. Through the analysis of voltage and current changes during the charging process, it can be found that constant current-constant voltage charging scheme guarantees the charging time and makes the battery work effectively. Then double closed-loop control method plays an important role in reducing the charging time and improving the charging efficiency. Besides, PFC obviously reduces the harmonic pollution caused by the charging process. So in the future development of electrical vehicles, constant current-constant voltage charging scheme and double closed-loop control will get greater development. Considering that the charging process may cause harmonic pollution to the electrical network, the combination of power factor correction device and charging device will be more and more popular.

\section{Acknowledgements}

This work is supported by the Key Project of NSFC (No. 61533012), the Shanghai Natural Science Foundation (14ZR1421800), the State Key Laboratory of Synthetical Automation for Process Industries.

\section{References}

[1] P. Guo, P. Liu, Research on development of electrical vehicles in china, IEEE International Conference on Future Information Technology and Management Engineering, (2015) 94-96 (in Chinese).

[2] K.M. Tsang, W.L. Chan, A simple and low-cost charger for lithium batteries, Journal of Power Sources. 191 (2009) 633-635. 
[3] S. Han, S. Han, S. Kaoru, Development of an optimal vehicle-to-grid aggregator for frequency regulation, IEEE Transactions on Smart Grid. 1 (2010) 65-72 (in Chinese).

[4] Y. Bao, J.C. Jiang, W.G. Zhang, A study on the on-board charging system for electrical vehicle based on SS-FB-PFC , Automotive Engineering. 33 (2011) 717-722 (in Chinese).

[5] J. Taylor, A. Maitra, M. Alexander, Evaluation of the impact of plug-in electrical vehicle loading on distribution system operations, Conference on Power \& Energy Society General Meeting, (2009) $1-6$. 